\title{
Extension complexity of stable set polytopes of bipartite graphs
}

\author{
Manuel Aprile, ${ }^{1}$ Yuri Faenza, ${ }^{2}$ Samuel Fiorini, ${ }^{3}$ Tony Huynh, ${ }^{3}$ Marco Macchia ${ }^{3}$ \\ 1 École Polytechnique Fédérale de Lausanne (EPFL), Lausanne, Switzerland \\ manuel.aprile@epfl.ch \\ ${ }^{2}$ IEOR Department, Columbia University, New York, USA yf2414@columbia.edu \\ 3 Université libre de Bruxelles, Brussels, Belgium \{sfiorini,mmacchia\}@ulb.ac.be \\ tony.bourbaki@gmail.com
}

\begin{abstract}
The extension complexity $\times \mathrm{c}(P)$ of a polytope $P$ is the minimum number of facets of a polytope that affinely projects to $P$. Let $G$ be a bipartite graph with $n$ vertices, $m$ edges, and no isolated vertices. Let $\operatorname{STAB}(G)$ be the convex hull of the stable sets of $G$. It is easy to see that $n \leqslant \mathrm{xc}(\operatorname{STAB}(G)) \leqslant n+m$. We improve both of these bounds. For the upper bound, we show that $\mathrm{xc}(\operatorname{STAB}(G))$ is $O\left(\frac{n^{2}}{\log n}\right)$, which is an improvement when $G$ has quadratically many edges. For the lower bound, we prove that $\mathrm{xc}(\operatorname{STAB}(G))$ is $\Omega(n \log n)$ when $G$ is the incidence graph of a finite projective plane. We also provide examples of 3-regular bipartite graphs $G$ such that the edge vs stable set matrix of $G$ has a fooling set of size $|E(G)|$.
\end{abstract}

\section{Introduction}

A polytope $Q \subseteq \mathbb{R}^{p}$ is an extension of a polytope $P \subseteq \mathbb{R}^{d}$ if there exists an affine map $\pi: \mathbb{R}^{p} \rightarrow \mathbb{R}^{d}$ with $\pi(Q)=P$. The extension complexity $\times \mathrm{c}(P)$ of $P$ is the minimum number of facets of any extension of $P$. If $Q$ is an extension of $P$ such that $Q$ has significantly fewer facets than $P$, then it is advantageous to run linear programming algorithms over $Q$ instead of $P$.

One example of a polytope that admits a much more compact representation in a higher dimensional space is the spanning tree polytope, $\mathrm{P}_{\text {sp.trees }}(G)$. Edmonds' [5] classic description of $\mathrm{P}_{\text {sp.trees }}(G)$ has $2^{\Omega(|V|)}$ facets. However, Wong [16] and Martin [11] proved that for every connected graph $G=(V, E)$,

$$
|E| \leqslant \mathrm{xc}\left(\mathrm{P}_{\text {sp.trees }}(G)\right) \leqslant O(|V| \cdot|E|) .
$$

Fiorini, Massar, Pokutta, Tiwary, and de Wolf [7] were the first to show that many polytopes arising from NP-hard problems (such as the stable set polytope) do indeed have high extension complexity. Their results answer an old question of Yannakakis [17] and do not rely on any complexity assumptions such as $\mathrm{P} \neq \mathrm{NP}$.

On the other hand, Rothvoß 12] proved that the perfect matching polytope of the complete graph $K_{n}$ has extension complexity at least $2^{\Omega(n)}$. This is somewhat surprising since the maximum weight matching problem can be solved in 
polynomial-time via Edmond's blossom algorithm [4. By now many accessible introductions to extension complexity are available (see [9, 1], 2], 13]).

Let $G=(V, E)$ be a (finite, simple) graph with $n:=|V|$ and $m:=|E|$. The stable set polytope of $G$, denoted $\operatorname{STAB}(G)$, is the convex hull of the characteristic vectors of stable sets of $G$. As previously mentioned, $\operatorname{STAB}(G)$ can have very high extension complexity. In 7 , it is proved that if $G$ is obtained from a complete graph by subdividing each edge twice, then $\mathrm{xc}(\operatorname{STAB}(G))$ is at least $2^{\Omega(\sqrt{n})}$. Very recently, Göös, Jain, and Watson 8 improved this to $2^{\Omega(n / \log n)}$, via a different class of graphs. For perfect graphs, Yannakakis [17 proved an upper bound of $n^{O(\log n)}$, and it is an open problem whether Yannakakis' upper bound can be improved to a polynomial bound.

In this paper we restrict our attention to bipartite graphs. Let $G=(V, E)$ be a bipartite graph with $n$ vertices, $m$ edges and no isolated vertices. By total unimodularity,

$$
\operatorname{STAB}(G)=\left\{x \in \mathbb{R}^{V} \mid x_{u} \geqslant 0 \text { for all } u \in V, x_{u}+x_{v} \leqslant 1 \text { for all } u v \in E\right\},
$$

and so $n \leqslant \mathrm{xc}(\operatorname{STAB}(G)) \leqslant n+m$. In this case $\mathrm{xc}(\operatorname{STAB}(G))$ lies in a very narrow range, and it is a good test of current methods to see if we can improve these bounds.

The situation is analogous to what happens with the spanning tree polytope of (arbitrary) graphs, where as previously mentioned, we also know that $\mathrm{xc}\left(\mathrm{P}_{\text {sp.trees }}(G)\right)$ lies in a very narrow range. Indeed, a notorious problem of Goemans (see [10]) is to improve the known bounds for $\mathrm{xc}\left(\mathrm{P}_{\text {sp.trees }}(G)\right)$, but this is still wide open.

However, for the stable set polytopes of bipartite graphs, we are able to give an improvement. Our main results are the following.

Theorem 1. For all bipartite graphs $G$ with $n$ vertices, the extension complexity of $\operatorname{STAB}(G)$ is $O\left(n^{2} / \log n\right)$.

Note that Theorem 1 is an improvement over the obvious upper bound when $G$ has quadratically many edges.

Theorem 2. There exists an infinite class $\mathcal{C}$ of bipartite graphs such that every $n$-vertex graph in $\mathcal{C}$ has extension complexity $\Omega(n \log n)$.

These are the first known examples of stable set polytopes of bipartite graphs where the extension complexity is more than linear in the number of vertices. For instance, $\mathrm{xc}\left(\operatorname{STAB}\left(K_{n / 2, n / 2}\right)\right)=\Theta(n)$. To the best of our knowledge, even for general perfect graphs $G$, the previous best lower bound for $\mathrm{xc}(\operatorname{STAB}(G))$ was the trivial bound $|V(G)|$.

Paper Organization. In Section 2 we define rectangle covers and fooling sets and we give examples of 3-regular graphs with tight fooling sets. We prove Theorem 1 in Section 3 and Theorem 2 in Section 4. In Section 5 we show that it is impossible to prove a better lower bound with the approach in Section 4 . Thus, to further improve the lower bound, different methods (or different graphs) are required. 


\section{Rectangle Covers and Fooling Sets}

Consider a polytope $P:=\operatorname{conv}(X)=\left\{x \in \mathbb{R}^{d} \mid A x \geqslant b\right\}$, where $X:=$ $\left\{x^{(1)}, \ldots, x^{(n)}\right\} \subseteq \mathbb{R}^{d}, A \in \mathbb{R}^{m \times d}$ and $b \in \mathbb{R}^{m}$. The slack matrix of $P$ (with respect to the chosen inner and outer descriptions of the polytope) is the matrix $S \in \mathbb{R}_{\geqslant 0}^{m \times n}$ having rows indexed by the inequalities $A_{1} x \geqslant b_{1}, \ldots, A_{m} x \geqslant b_{m}$ and columns indexed by the points $x^{(1)}, \ldots, x^{(n)}$, defined as $S_{i j}:=A_{i} x^{(j)}-b_{i} \geqslant 0$.

Yannakakis [17] proved that the extension complexity of $P$ equals the nonnegative rank of $S$. In this work, we only rely on a lower bound that follows directly from this fact. For a matrix $M$, we define the support of $M$ as $\operatorname{supp}(M):=\left\{(i, j) \mid M_{i j} \neq 0\right\}$. A rectangle is any set of the form $R=I \times J$, with $R \subseteq \operatorname{supp}(M)$. A size- $k$ rectangle cover of $M$ is a collection $R_{1}, \ldots, R_{k}$ of rectangles such that $\operatorname{supp}(M)=R_{1} \cup \cdots \cup R_{k}$. The rectangle covering bound of $M$ is the minimum size of a rectangle cover of $M$, and is denoted $\operatorname{rc}(M)$.

Theorem 3 (Yannakakis, [17]). Let $P$ be a polytope with $\operatorname{dim}(P) \geqslant 1$ and let $S$ be any slack matrix of $P$. Then, $\operatorname{xc}(P) \geqslant \operatorname{rc}(S)$.

A fooling set for $M$ is a set of entries $F \subseteq \operatorname{supp}(M)$ such that $M_{i \ell} \cdot M_{k j}=0$ for all distinct $(i, j),(k, \ell) \in F$. The largest size of a fooling set of $M$ is denoted by fool $(M)$. Clearly, $\mathrm{rc}(M) \geqslant$ fool $(M)$.

Let $G$ be a bipartite graph. The edge vs stable set matrix of $G$, denoted $M(G)$, is the $0 / 1$ matrix with a row for each edge of $G$, a column for each stable set of $G$, and a 1 in position $(e, S)$ if and only if $e \cap S=\varnothing$ (as usual, we regard edges as pairs of vertices). We say that $G$ has a tight fooling set if $M(G)$ has a fooling set of size $|E(G)|$. Note that if $G$ has a tight fooling set, then the non-negative rank of $M(G)$ is exactly $|E(G)|$. Also observe that the property of having a tight fooling set is closed under taking subgraphs.

It is easy to check that even cycles have tight fooling sets. We now give an infinite family of 3-regular graphs that have tight fooling sets. A graph is $C_{4}$-free if it does not contain a cycle of length four.

Theorem 4. Let $G=(V, E)$ be a 3 -regular, $C_{4}$-free bipartite graph. Then $G$ has a tight fooling set.

Proof. For $X \subseteq V$, we let $N(X)$ denote the set of neighbours of $X$. Let $V=A \cup B$ be a bipartition of the vertex set, and let $\phi: E \rightarrow\{1,2,3\}$ be a proper edge coloring of $G$, which exists by 3-regularity and König's edge-coloring theorem (see e.g. [14, Theorem 20.1]). For each vertex $a \in A$, we name its neighbors $a_{1}, a_{2}, a_{3} \in B$ so that $\phi\left(a a_{i}\right)=i$. For each $a \in A$, consider the following stable sets:

$$
\begin{aligned}
& S_{a a_{1}}:=A \backslash\{a\} \\
& S_{a a_{2}}:=\left\{a_{1}\right\} \cup\left\{a^{\prime} \in A \mid a^{\prime} \notin N\left(a_{1}\right)\right\} \\
& S_{a a_{3}}:=B \backslash\left\{a_{3}\right\} .
\end{aligned}
$$

This defines a stable set $S_{e}$ disjoint from $e$, for every edge $e \in E$. Since $\phi$ is proper, no two of these stable sets are equal. We claim that $\left\{\left(e, S_{e}\right) \mid e \in E\right\}$ is a fooling set in the edge vs stable set matrix of $G$. 
Let $e$ and $f$ be distinct edges. We want to show that $S_{e}$ intersects $f$ or $S_{f}$ intersects $e$. Consider the following three cases. Let $e=a a_{i}$, where $i=\phi(e)$.

Case 1. If $\phi(e)=1$, then $S_{e}=S_{a a_{1}}$ intersects $f$ unless $f=a a_{i}$ for some $i \in\{2,3\}$. In both cases we have $a_{1} \in S_{f} \cap e$.

Case 2. If $\phi(e)=3$, then $S_{e}=S_{a a_{3}}$ intersects $f$ unless $f=a^{\prime} a_{3}$ for some $a^{\prime} \in A$. Either $\phi(f)=1$ and $S_{f}$ intersects $e$ (as in Case 1), or $\phi(f)=2$. In the last case, since $G$ is $C_{4}$-free, we have $a \notin N\left(a_{1}^{\prime}\right)$. It follows that $S_{f}=S_{a^{\prime} a_{3}}=S_{a^{\prime} a_{2}^{\prime}}$ intersects $e$.

Case 3. If $\phi(e)=2$, then we may also assume $\phi(f)=2$ since otherwise by exchanging the roles of $e$ and $f$ we are back to one of the previous cases. Let $a^{\prime}$ denote the endpoint of $f$ in $A$, so that $f=a^{\prime} a_{2}^{\prime}$. Because $\phi$ is proper, $a^{\prime} \neq a$ and $a_{1}^{\prime} \neq a_{1}$. Since $G$ is $C_{4}$-free, we have $a \notin N\left(a_{1}^{\prime}\right)$ or $a^{\prime} \notin N\left(a_{1}\right)$. Hence, $a \in S_{f} \cap e$ or $a^{\prime} \in S_{e} \cap f$.

Note that there are infinitely many 3 -regular, $C_{4}$-free bipartite graphs. For example, we can take a hexagonal grid on a torus.

\section{An Improved Upper Bound}

In this section we prove Theorem 1, We use the following result of Martin [11.

Lemma 5. If $Q$ is a nonempty polyhedron, $\gamma \in \mathbb{R}$, and

$$
P=\{x \mid\langle x, y\rangle \leqslant \gamma \text { for every } y \in Q\},
$$

then $\mathrm{xc}(P) \leqslant \mathrm{xc}(Q)+1$.

The edge polytope $\mathrm{P}_{\text {edge }}(G)$ of a graph $G$ is the convex hull of the incidence vectors in $\mathbb{R}^{V(G)}$ of all edges of $G$. The second ingredient we need is the following bound on the extension complexity of the edge polytope of all $n$-vertex graphs due to Fiorini, Kaibel, Pashkovich, and Theis [6, Lemma 3.4]. This bound follows from a nice result of Tuza [15, which states that every $n$-vertex graph can be covered with a set of bicliques of total weight $O\left(n^{2} / \log n\right)$, where the weight of a biclique is its number of vertices.

Lemma 6. For every graph $G$ with $n$ vertices, $\mathrm{xc}\left(\mathrm{P}_{\text {edge }}(G)\right)=O\left(n^{2} / \log n\right)$.

Proof of Theorem 1, Let $G=(V, E)$. Since

$$
\operatorname{STAB}(G)=\mathbb{R}_{\geqslant 0}^{V} \cap\left\{x \in \mathbb{R}^{V} \mid\langle x, y\rangle \leqslant 1 \text { for every } y \in \operatorname{P}_{\text {edge }}(G)\right\},
$$

By Lemmas 5 and 6, the extension complexity of $\operatorname{STAB}(G)$ is $O\left(n^{2} / \log n\right)$. 


\section{An Improved Lower Bound}

In this section we prove Theorem 2 The examples we use to prove our lower bound are incidence graphs of finite projective planes. We will not use any theorems from projective geometry, but the interested reader can refer to [3].

Let $q$ be a prime power, $\operatorname{GF}(q)$ be the field with $q$ elements, and $\mathrm{PG}(2, q)$ be the projective plane over $\mathrm{GF}(q)$. The incidence graph of $\mathrm{PG}(2, q)$, denoted $\mathcal{I}(q)$, is the bipartite graph with bipartition $(\mathcal{P}, \mathcal{L})$, where $\mathcal{P}$ is the set of points of $\mathrm{PG}(2, q), \mathcal{L}$ is the set of lines of $\mathrm{PG}(2, q)$, and $\mathrm{p} \in \mathcal{P}$ is adjacent to $\ell \in \mathcal{L}$ if and only if the point $\mathrm{p}$ lies on the line $\ell$. For example, $\mathrm{PG}(2,2)$ and its incidence graph $\mathcal{I}(2)$ are depicted in Figure 1 .

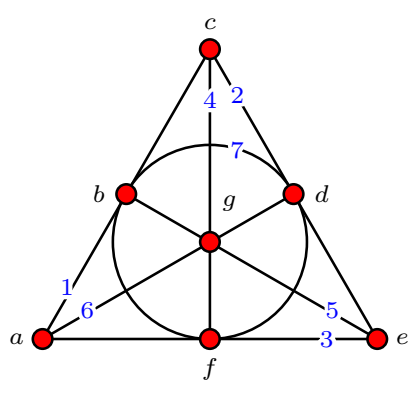

(a)

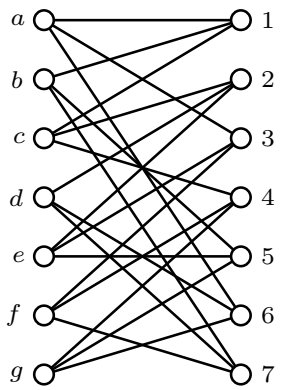

(b)

Fig. 1. $P G(2,2)$ and its incidence graph $\mathcal{I}(2)$.

Before proving Theorem 2 we gather a few lemmas on binomial coefficients. The first two are well-known, so we omit the easy proofs.

Lemma 7. For all integers $h$ and $c$ with $h \geqslant c \geqslant 0$

$$
\sum_{j=c}^{h}\left(\begin{array}{l}
j \\
c
\end{array}\right)=\left(\begin{array}{l}
h+1 \\
c+1
\end{array}\right) .
$$

Lemma 8. For all positive integers $x, y$, and $h$,

$$
\sum_{j=0}^{h}\left(\begin{array}{c}
x+j \\
j
\end{array}\right)\left(\begin{array}{c}
h+y-j \\
h-j
\end{array}\right)=\left(\begin{array}{c}
x+y+h+1 \\
h
\end{array}\right) .
$$

Lemma 9. Let $q, c, t$ be positive integers with $c+t \leqslant q+1$. Then

$$
t \sum_{k=c}^{q+1-t} \frac{1}{k}\left(\begin{array}{c}
q+1-t-c \\
k-c
\end{array}\right)\left(\begin{array}{l}
q \\
k
\end{array}\right)^{-1}=\left(\begin{array}{c}
t+c-1 \\
t
\end{array}\right)^{-1} \leqslant \frac{1}{c} .
$$


Proof. We have that

$$
\begin{aligned}
& t \sum_{k=c}^{q+1-t} \frac{1}{k}\left(\begin{array}{c}
q+1-t-c \\
k-c
\end{array}\right)\left(\begin{array}{l}
q \\
k
\end{array}\right)^{-1} \\
= & \frac{t(q+1-t-c) !}{q !} \sum_{k=c}^{q+1-t} \frac{(k-1) !(q-k) !}{(k-c) !(q+1-t-k) !} \\
= & \frac{t(q+1-t-c) !}{q !}(c-1) !(t-1) ! \sum_{k=c}^{q+1-t}\left(\begin{array}{l}
k-1 \\
c-1
\end{array}\right)\left(\begin{array}{l}
q-k \\
t-1
\end{array}\right) .
\end{aligned}
$$

Moreover,

$$
\begin{aligned}
\sum_{k=c}^{q+1-t}\left(\begin{array}{c}
k-1 \\
c-1
\end{array}\right)\left(\begin{array}{c}
q-k \\
t-1
\end{array}\right) & =\sum_{j=0}^{q+1-t-c}\left(\begin{array}{c}
c-1+j \\
c-1
\end{array}\right)\left(\begin{array}{c}
q-c-j \\
t-1
\end{array}\right) \\
{[h=q+1-t-c, x=c-1, y=t-1] } & =\sum_{j=0}^{h}\left(\begin{array}{c}
x+j \\
j
\end{array}\right)\left(\begin{array}{c}
h+y-j \\
h-j
\end{array}\right) \\
\text { [by Lemma } 8] & =\left(\begin{array}{c}
x+y+h+1 \\
h
\end{array}\right) \\
& =\left(\begin{array}{c}
q \\
q+1-t-c
\end{array}\right) .
\end{aligned}
$$

We conclude that

$$
\begin{aligned}
t \sum_{k=c}^{q+1-t} \frac{1}{k}\left(\begin{array}{c}
q+1-t-c \\
k-c
\end{array}\right)\left(\begin{array}{l}
q \\
k
\end{array}\right)^{-1} & =\frac{t(q+1-t-c) !}{q !} \frac{q !(c-1) !(t-1) !}{(q+1-t-c) !(t+c-1) !} \\
& =\left(\begin{array}{c}
t+c-1 \\
t
\end{array}\right)^{-1} .
\end{aligned}
$$

The number of $t$-subsets of a set of size $t+c-1$ is at least $c$, since it includes all $t$-subsets containing a fixed set of size $t-1$. Hence, $\left(\begin{array}{c}t+c-1 \\ t\end{array}\right)^{-1} \leqslant \frac{1}{c}$.

From the definition of $\mathrm{PG}(2, q)$ it follows that that $\mathcal{I}(q)$ is $(q+1)$-regular, $|V(\mathcal{I}(q))|=2\left(q^{2}+q+1\right)$, and $|E(\mathcal{I}(q))|=(q+1)\left(q^{2}+q+1\right)$. Let $n=q^{2}+q+1$ and note that $\mathcal{I}(q)$ has $2 n$ vertices. We let $\mathcal{P}$ and $\mathcal{L}$ denote the set of points and lines of PG $(2, q)$. We also use the fact that $\mathcal{I}(q)$ is $C_{4}$-free.

We denote the edge vs stable set incidence matrix of $\mathcal{I}(q)$ by $S_{q}$. Each 1-entry of $S_{q}$ is of the form $(e, S)$ where $e \in E, S \subseteq V$ is a stable set, and $e \cap S=\varnothing$. To prove Theorem 2 we will assign weights to the 1-entries of $S_{q}$ in such a way that the total weight is at least $\Omega(n \log n)$, while the weight of every rectangle is at most 1 . The only entries that will receive non-zero weight are what we call special entries, which we now define.

Definition 10. A 1-entry of $S_{q}$ is special if it has the form $(e, S(X))$ where 
- $e=\mathrm{p} \ell$ with $\mathrm{p} \in \mathcal{P}, \ell \in \mathcal{L}$,

- $X \subseteq N(\ell) \backslash\{\mathrm{p}\}, X$ non-empty,

- $S(X)=X \cup(\mathcal{L} \backslash N(X))$.

We also need the following compact representation of maximal rectangles.

Definition 11. Let $R$ be a maximal rectangle. Then $R$ is determined by a pair $\left(\mathcal{P}_{R}, \mathcal{L}_{R}\right)$ with $\mathcal{P}_{R} \subseteq \mathcal{P}, \mathcal{L}_{R} \subseteq \mathcal{L}$, where the rows of $R$ are all the edges between $\mathcal{P}_{R}$ and $\mathcal{L}_{R}$ and the columns of $R$ are all the stable sets $S \subseteq V \backslash\left(\mathcal{P}_{R} \cup \mathcal{L}_{R}\right)$.

We are now ready to prove Theorem 2 in the following form.

Theorem 2, Let $q$ be a prime power and $n=q^{2}+q+1$. Then there exists a constant $c>0$ such that

$$
\mathrm{xc}(\operatorname{STAB}(\mathcal{I}(q))) \geqslant c n \log n .
$$

Proof. Let $n=q^{2}+q+1$. Let $V=\mathcal{P} \cup \mathcal{L}$ be the vertices of $\mathcal{I}(q)$, and $E$ be the edges of $\mathcal{I}(q)$. To each special entry $(e, S(X))$ we assign the weight

$$
w(e, S(X))=\frac{1}{|X|\left(\begin{array}{c}
q \\
|X|
\end{array}\right)(q+1)} .
$$

All other entries of $S_{q}$ receive weight zero.

Claim 12. $w\left(S_{q}\right):=\sum_{(e, S)} w(e, S) \geqslant c n \log n$ for some constant $c$.

Subproof. We have that

$$
\begin{aligned}
\sum_{(e, S)} w(e, S)=\sum_{(e, S(X)) \text { special }} w(e, S(X)) & =\sum_{e \in E} \sum_{k=1}^{q}\left(\begin{array}{l}
q \\
k
\end{array}\right) \frac{1}{k\left(\begin{array}{l}
q \\
k
\end{array}\right)(q+1)} \\
& =\frac{|E|}{q+1} \sum_{k=1}^{q} \frac{1}{k}=n \sum_{k=1}^{q} \frac{1}{k}>c n \log n .
\end{aligned}
$$

The claim follows.

Let $R=\left(\mathcal{P}_{R}, \mathcal{L}_{R}\right)$ be an arbitrary maximal rectangle. We finish the proof by showing that $w(R):=\sum_{(e, S) \in R} w(e, S) \leqslant 1$. Together with Claim 12 this clearly implies Theorem 2, We will need the following obvious but useful Claim.

Claim 13. A special entry $(\mathrm{p} \ell, S(X))$ is covered by $R=\left(\mathcal{P}_{R}, \mathcal{L}_{R}\right)$ if and only if $X \cap \mathcal{P}_{R}=\varnothing, \mathcal{L}_{R} \subseteq N(X), \mathrm{p} \in \mathcal{P}_{R}$, and $\ell \in \mathcal{L}_{R}$.

We consider two cases. First suppose that $\mathcal{L}_{R}=\{\ell\}$ for some $\ell$. Then the only special entries covered by $R$ are of the form (p $\ell, S(X)$ ), with $X \subseteq N(\ell) \backslash \mathcal{P}_{R}$. Let $N(\ell) \cap \mathcal{P}_{R}=\left\{\mathrm{p}_{1}, \ldots, \mathrm{p}_{t}\right\}$, where $1 \leqslant t \leqslant q+1$. To compute $w(R)$ we have 
to sum over all edges $\mathrm{p}_{i} \ell$ and over all subsets $X \subseteq N(\ell) \backslash\left\{\mathrm{p}_{1}, \ldots, \mathrm{p}_{t}\right\}$. It follows that

$$
\begin{aligned}
w(R) & =\sum_{i=1}^{t} \sum_{k=1}^{q+1-t}\left(\begin{array}{c}
q+1-t \\
k
\end{array}\right) \frac{1}{k\left(\begin{array}{l}
q \\
k
\end{array}\right)(q+1)} \\
& =t \sum_{k=1}^{q+1-t} \frac{(q+1-t) !}{k !(q+1-t-k) !} \frac{k !(q-k) !}{k q !(q+1)} \\
& =\frac{t(q+1-t) !(t-1) !}{(q+1) !} \sum_{k=1}^{q+1-t}\left(\begin{array}{c}
q-k \\
q+1-t-k
\end{array}\right) \frac{1}{k} \\
& =\frac{1}{\left(\begin{array}{c}
q+1 \\
t
\end{array}\right)} \sum_{k=1}^{q+1-t}\left(\begin{array}{c}
q-k \\
t-1
\end{array}\right) \frac{1}{k} \leqslant \frac{1}{\left(\begin{array}{c}
q+1 \\
t
\end{array}\right)} \sum_{j=t-1}^{q-1}\left(\begin{array}{c}
j \\
t-1
\end{array}\right)=\frac{1}{\left(\begin{array}{c}
q+1 \\
t
\end{array}\right)}\left(\begin{array}{l}
q \\
t
\end{array}\right) \leqslant 1,
\end{aligned}
$$

where the last equality follows from Lemma 7 ,

The remaining case is if $\left|\mathcal{L}_{R}\right| \geqslant 2$. For $\ell \in \mathcal{L}_{R}$ such that $(\mathrm{p} \ell, S(X))$ is covered by $R$ for some $\mathrm{p}, X$, define

$k_{\ell}=\min \{|X| \mid$ there exist $\mathrm{p}, X:(\mathrm{p} \ell, S(X))$ is a special entry covered by $R\}$.

Claim 14. Let $(\mathrm{p} \ell, S(X))$ be a special entry covered by $R$ such that $|X|=k_{\ell}$. Then for each $\mathrm{p}^{\prime}, Y$ such that $R$ covers $\left(\mathrm{p}^{\prime} \ell, S(Y)\right)$, we have $X \subseteq Y$.

Subproof. For each $\ell^{\prime} \in \mathcal{L}_{R} \backslash\{\ell\}$ (there is at least one since $\left|\mathcal{L}_{R}\right|>1$ ), we have $\ell^{\prime} \in N(X)$ by Claim 13. That is, there is $\mathrm{x}=\mathrm{x}\left(\ell^{\prime}\right) \in X$ adjacent to $\ell^{\prime}$. Similarly, since $\ell^{\prime} \in N(Y)$, there is $\mathrm{y}=\mathrm{y}\left(\ell^{\prime}\right) \in Y$ adjacent to $\ell^{\prime}$. Now, if $\mathrm{x}\left(\ell^{\prime}\right) \neq \mathrm{y}\left(\ell^{\prime}\right)$, then $\mathcal{I}(q)$ contains a 4-cycle, which is a contradiction. Hence we must have $\mathrm{x}\left(\ell^{\prime}\right)=\mathrm{y}\left(\ell^{\prime}\right)$ for all $\ell^{\prime} \in \mathcal{L}_{R} \backslash\{\ell\}$. Now if there is an $\mathrm{x} \in X$ such that $\mathrm{x} \neq \mathrm{x}\left(\ell^{\prime}\right)$ for every $\ell^{\prime} \in \mathcal{L}_{R} \backslash\{\ell\}$, then $(\mathrm{p} \ell, S(X \backslash\{\mathrm{x}\}))$ is still covered by $R$, contradicting the minimality of $X$. We conclude $X \subseteq Y$, as required.

Now fix $\ell \in \mathcal{L}_{R}$, and let

$$
w(\ell)=\sum\{w(\mathrm{p} \ell, S(X)) \mid(\mathrm{p} \ell, S(X)) \text { special }\} .
$$

Claim 15. For every $\ell \in \mathcal{L}_{R}$,

$$
w(\ell) \leqslant \frac{1}{(q+1) k_{\ell}} .
$$

Subproof. Let $N(\ell) \cap \mathcal{P}_{R}=\left\{\mathrm{p}_{1}, \ldots, \mathrm{p}_{t}\right\}$, where $1 \leqslant t \leqslant q+1$. Let $X$ be such that $(\mathrm{p} \ell, S(X))$ is a special entry covered by $R$ and $|X|=k_{\ell}$. By Claim 14, the only special entries appearing in the above sum are of the form $\left(\mathrm{p}_{i} \ell, S(Y)\right)$ where $i \in[t]$ and $X \subseteq Y \subseteq\left(\mathcal{P} \backslash \mathcal{P}_{R}\right) \cap N(\ell)$. Therefore

$$
w(\ell) \leqslant t \sum_{k=k_{\ell}}^{q+1-t}\left(\begin{array}{c}
q+1-t-k_{\ell} \\
k-k_{\ell}
\end{array}\right) \frac{1}{k\left(\begin{array}{l}
q \\
k
\end{array}\right)(q+1)} \leqslant \frac{1}{(q+1) k_{\ell}},
$$

where the last inequality follows from Lemma 9 with $c=k_{\ell}$. 
Claim 16. For every $\ell \in \mathcal{L}_{R},\left|\mathcal{L}_{R}\right| \leqslant(q+1) k_{\ell}$.

Subproof. Again, let $X$ be such that $(\mathrm{p} \ell, S(X))$ is covered by $R$ and assume that $|X|=k_{\ell}$. By Claim [13, we have $\mathcal{L}_{R} \subseteq N(X)$.

Hence $\left|\mathcal{L}_{R}\right| \leqslant|N(X)| \leqslant(q+1)|X|=(q+1) k_{\ell}$.

By Claim 15 and Claim [16] for every $\ell \in \mathcal{L}_{R}, w(\ell) \leqslant \frac{1}{\mid \mathcal{L}_{R}}$. But clearly $w(R)=\sum_{\ell \in \mathcal{L}_{R}} w(\ell)$, and so $w(R) \leqslant 1$, as required. This completes the entire proof.

\section{A small rectangle cover of the special entries}

In this section we show that the submatrix of special entries considered in the previous section has a rectangle cover of size $O(n \log n)$. Combined with Theorem 2. this implies that a minimal set of rectangles that cover all the special entries always has size $\Theta(n \log n)$. Thus, to improve our bound, we must consider a different set of entries of the slack matrix, or use a different set of graphs.

This cover will be built from certain labeled trees which we now define. Note that the length of a path is its number of edges.

Definition 17. For every integer $k \geqslant 1$, we build a tree $T(k)$ recursively:

- The tree $T(1)$ consists of a root $r$ and a single leaf attached to it.

- For $k>1$, we construct $T(k)$ by first identifying one end of a path $P_{1}$ of length $k_{1}:=\left\lceil\frac{k}{2}\right\rceil$ to another end of a path $P_{2}$ of length $k_{2}:=\left\lfloor\frac{k}{2}\right\rfloor$ along a root vertex $r$. Let $\lambda_{i}$ be the end of $P_{i}$ that is not $r$. We then attach a copy of $T\left(k_{i}\right)$ to $\lambda_{3-i}$, identifying $\lambda_{3-i}$ with the root of $T\left(k_{i}\right)$. We call $P_{1}$ and $P_{2}$ the main paths of $T(k)$.

The next Lemma follows easily by induction on $k$.

Lemma 18. For all $k \geqslant 1$,

(i) $T(k)$ has $O(k \log k)$ vertices;

(ii) $T(k)$ has $k$ leaves;

(iii) every path from the root $r$ to a leaf has length $k$.

Definition 19. We recursively define a labeling $\varphi_{k}: V(T(k)) \backslash\{r\} \rightarrow[k]$ as follows:

- Let $v$ be the non-root vertex of $V(T(1))$ and set $\varphi_{1}(v):=1$.

- For $k>1$, let $P_{1}$ and $P_{2}$ be the main paths of $T(k)$. We name the vertices of $P_{1}$ as $r, v_{1}, \ldots, v_{\left\lceil\frac{k}{2}\right\rceil}$ and $P_{2}$ as $r, v_{\left\lceil\frac{k}{2}\right\rceil+1}, \ldots, v_{k}$, where these vertices are listed according to their order along $P_{1}$ and $P_{2}$. Set $k_{1}:=\left\lceil\frac{k}{2}\right\rceil$ and $k_{2}:=\left\lfloor\frac{k}{2}\right\rfloor$. Note that $V(T(k))=\bigcup_{i=1,2}\left(V\left(P_{i}\right) \cup V\left(B_{i}\right)\right)$, where $B_{i}$ is a copy of the tree $T\left(k_{3-i}\right)$. We define 


$$
\varphi_{k}(v)= \begin{cases}i, & \text { if } v=v_{i} \\ \varphi_{k_{2}}(v)+k_{1}, & \text { if } v \in V\left(B_{1}\right) \backslash V\left(P_{1}\right) \\ \varphi_{k_{1}}(v), & \text { if } v \in V\left(B_{2}\right) \backslash V\left(P_{2}\right)\end{cases}
$$

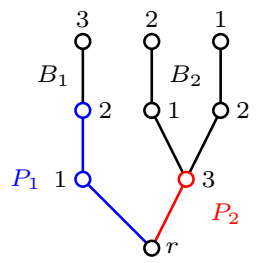

(a) $T(3)$ and the labeling $\varphi_{3}$

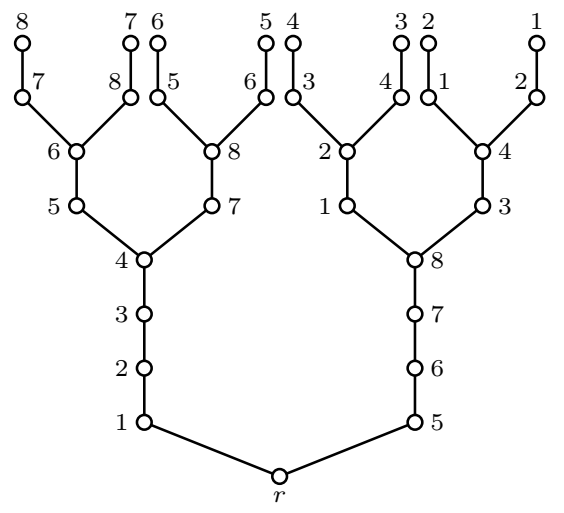

(b) $T(8)$ and the labeling $\varphi_{8}$

Fig. 2.

For each vertex $v \in T(k)$ we let $P(v)$ be the path in $T(k)$ from $r$ to $v$.

Lemma 20. Let $\varphi_{k}, B_{1}$, and $B_{2}$ be as in Definition 19.

(i) If $L$ is the set of leaves of $T(k)$, then $\varphi_{k}\left(L \cap V\left(B_{1}\right)\right)=\left\{\left\lceil\frac{k}{2}\right\rceil+1, \ldots, k\right\}$ and $\varphi_{k}\left(L \cap V\left(B_{2}\right)\right)=\left\{1, \ldots,\left\lceil\frac{k}{2}\right\rceil\right\}$.

(ii) For every leaf $\lambda$ of $T(k), \varphi_{k}(V(P(\lambda)) \backslash\{r\})=[k]$.

(iii) Each label $i \in[k]$ occurs at most $\lceil\log k\rceil+1$ times in the labeling of $T(k)$.

Proof. We proceed by induction on $k$. Property (i) follows directly from the recursive definition of the labeling $\varphi_{k}$.

For (ii) let $\lambda$ be a leaf and let the (ordered) vertices of $P(\lambda)$ be $r, p_{1}, \ldots, p_{k}=$ $\lambda$. Suppose that $\lambda \in V\left(B_{i}\right)$. Then $P(\lambda):=P_{i} \cup P^{\prime}$, where $P_{i}$ is a main path of $T(k)$ and $P^{\prime}$ is the path in $B_{i}$ going from the root of $B_{i}$ to $\lambda$. Property (ii) now follows by induction and the definition of $\varphi_{k}$.

For (iii), first suppose that the label $i$ is in $\left[k_{1}\right]$. Then $i$ appears exactly once in the labeling of the main path $P_{1}$ of $T(k)$, it does not figure in the labeling of the nodes $V\left(P_{2}\right) \cup\left(V\left(B_{1}\right) \backslash V\left(P_{1}\right)\right)$, and, by the inductive step, it occurs $\left\lceil\log \left\lceil\frac{k}{2}\right\rceil\right\rceil+1=\lceil\log k\rceil$ times in $\varphi_{k}\left(B_{2}\right)$. The thesis follows. A similar argument settles the remaining case $i \in[k] \backslash\left[k_{1}\right]$.

Henceforth, we simplify notation and denote the labeling $\varphi_{k}$ of $T(k)$ as $\varphi$. We now recall some notation from the previous section. Let $q$ be a prime power and $S_{q}$ be the edge vs stable set incidence matrix of $\mathcal{I}(q)$. 
A maximal rectangle $R=\left(\mathcal{P}_{R}, \mathcal{L}_{R}\right)$ is centered if $\left|\mathcal{L}_{R}\right| \geqslant 2$ and there is a point $\mathrm{c} \in \mathcal{P} \backslash \mathcal{P}_{R}$ such that $\mathrm{c}$ is incident to all lines in $\mathcal{L}_{R}$. We call c the center of $R$. Note that the center is unique and its existence implies that $\left|\mathcal{L}_{R}\right| \leqslant q+1$.

One way to create centered rectangles is as follows. Let $\ell$ be a line, $\mathrm{c}$ be a point on $\ell$, and $Y \subseteq N(\ell)$ with $\mathrm{c} \in Y$. We let $\mathrm{c}, \ell, Y$ be the centered rectangle $R=\left(\mathcal{P}_{R}, \mathcal{L}_{R}\right)$ where $\mathcal{P}_{R}=N(\ell) \backslash Y$ and $\mathcal{L}_{R}=N(\mathrm{c})$. Note that a special entry of the form $(\mathrm{p} \ell, S(X))$ is covered by the centered rectangle $\mathrm{c}, \ell, Y$ if and only if $\mathrm{p} \notin Y$ and $\mathrm{c} \in X \subseteq Y$.

We now fix a line $\ell \in \mathrm{PG}(2, q)$ and let $N(\ell)=\left\{\mathrm{p}_{1}, \ldots, \mathrm{p}_{q+1}\right\}$. We will use the labeling $\varphi$ of $T(q+1)$ to provide a collection of centered rectangles that cover all special entries of the form $(\mathrm{p} \ell, S(X))$. Recall that for a vertex $v$ of $T(q+1)$, $P(v)$ denotes the path in $T(q+1)$ from $r$ to $v$. If $v$ is neither the root nor a leaf of $T(q+1)$, we define

$$
Y(v):=\left\{\mathrm{p}_{\varphi(u)} \mid u \text { is a non-root vertex of } P(v)\right\} .
$$

Lemma 21. Fix a line $\ell \in \mathrm{PG}(2, q)$ and let $N(\ell)=\left\{\mathrm{p}_{1}, \ldots, \mathrm{p}_{q+1}\right\}$. Let $\mathcal{R}_{\ell}$ be the collection of all centered rectangles $\mathrm{p}_{\varphi(v)}, \ell, Y(v)$ where $v$ ranges over all non-root, non-leaf vertices of $T(q+1)$. Then every special entry $(e, S)$ with $\ell$ incident to $e$ is covered by some rectangle $R \in \mathcal{R}_{\ell}$.

Proof. Let $\left(\mathrm{p}_{i} \ell, S(X)\right)$ be such a special entry and let $\lambda$ be the (unique) leaf of $T(q+1)$ such that $\varphi(\lambda)=i$. Name the vertices of $P(\lambda)$ as $r, u_{1}, \ldots, u_{q+1}=\lambda$ (ordered away from the root).

Define $j=\max \left\{i \mid \mathrm{p}_{\varphi\left(u_{i}\right)} \in X\right\}$. Since $\mathrm{p}_{\varphi(\lambda)} \notin X$, note $j<q+1$. By Lemma 20. $X \subseteq Y\left(u_{j}\right)$. Also, by construction, $\mathrm{p}_{\varphi\left(u_{j}\right)} \in X$ and $\mathrm{p} \notin Y\left(u_{j}\right)$. We conclude that the centered rectangle $\mathrm{p}_{\varphi\left(u_{j}\right)}, \ell, Y\left(u_{j}\right)$ covers the special entry $\left(\mathrm{p}_{i} \ell, S(X)\right)$, as required.

By Lemma 21 for each line $\ell$, there is a set $\mathcal{R}_{\ell}$ of $O(q \log q)$ centered rectangles that cover all special entries of the form $(\mathrm{p} \ell, S(X))$. By taking the union of all $\mathcal{R}_{\ell}$, we get a cover $\mathcal{R}$ of size $O(n q \log q)$ for all the special entries. To prove the main theorem of this section, we now reduce the size of $\mathcal{R}$ by a factor of $q$.

Theorem 22. There is a set of $O(n \log n)$ centered rectangles that cover all the special entries.

Proof. If $R_{1}:=\mathrm{c}, \ell_{1}, Y_{1}, \ldots, R_{k}:=\mathrm{c}, \ell_{k}, Y_{k}$ are centered rectangles with the same center c, we let $\sum_{i=1}^{k} R_{i}=R$ be the maximal rectangle with $\mathcal{P}_{R}=$ $\bigcup_{i=1}^{k} N\left(\ell_{i}\right) \backslash \bigcup_{i=1}^{k} Y_{i}$ and $\mathcal{L}_{R}=N(\mathrm{c})$. Note that $\sum_{i=1}^{k} R_{i}$ is also a centered rectangle with center $\mathrm{c}$.

Claim 23. If $R_{1}:=\mathrm{c}, \ell_{1}, Y_{1}, \ldots, R_{k}:=\mathrm{c}, \ell_{k}, Y_{k}$ are centered rectangles such that $\ell_{1}, \ldots, \ell_{k}$ are all distinct, then $\sum_{i=1}^{k} R_{i}$ covers all special entries covered by $\bigcup_{i=1}^{k} R_{i}$. 
Subproof. Let $(\mathrm{p} \ell, S(X))$ be a special entry covered by some $\mathrm{c}, \ell_{j}, Y_{j}$. Clearly $\mathrm{c} \in X \subseteq Y_{j} \subseteq \bigcup_{i=1}^{k} Y_{i}$. By contradiction, suppose $\mathrm{p} \in \bigcup_{i=1}^{k} Y_{i}$. Since $\mathrm{p} \notin Y_{j}$, $\mathrm{p} \in Y_{j^{\prime}} \subseteq N\left(\ell_{j^{\prime}}\right)$ for some $j^{\prime} \neq j$. But then $\mathrm{c} \ell_{j} \mathrm{p} \ell_{j^{\prime}}$ is a 4-cycle in $\mathcal{I}(q)$, which is a contradiction. Hence the entry $(\mathrm{p} \ell, S(X))$ is also covered by $\sum_{i=1}^{k} R_{i}$.

We iteratively use Claim 23 to reduce the number of rectangles in our covering $\mathcal{R}$. For each point c, name the $q+1$ lines through c as $\ell, \ell_{1}, \ldots, \ell_{q}$, so that among $\mathcal{R}_{\ell}, \mathcal{R}_{\ell_{1}}, \ldots, \mathcal{R}_{\ell_{q}}$, the collection $\mathcal{R}_{\ell}$ has the most rectangles with center c. Note that, by Lemma 20, $\mathcal{R}_{\ell}$ contains $O(\log q)$ rectangles with center c.

Fix $i \in[q]$ and for each rectangle $R \in \mathcal{R}_{\ell_{i}}$ with center c choose a rectangle $f_{i}(R)$ with center c in $\mathcal{R}_{\ell}$ such that $f_{i}(R) \neq f_{i}\left(R^{\prime}\right)$ if $R \neq R^{\prime}$. For each $R \in \mathcal{R}_{\ell}$ we let

$$
f^{-1}(R)=\{R\} \cup \bigcup_{i=1}^{q}\left\{R^{\prime} \in \mathcal{R}_{\ell_{i}} \mid f_{i}\left(R^{\prime}\right)=R\right\} .
$$

We then remove all rectangles with center c that appear in $\mathcal{R}_{\ell}, \mathcal{R}_{\ell_{1}}, \ldots, \mathcal{R}_{\ell_{q}}$ and replace them with all rectangles of the form $\sum_{R^{\prime} \in f^{-1}(R)} R^{\prime}$, where $R$ ranges over all rectangles in $\mathcal{R}_{\ell}$ with center c. In doing so, we obtain at most $O(\log q)=$ $O(\log n)$ rectangles with center c. Repeating for every c $\in \mathcal{P}$ gives us $O(n \log n)$ rectangles in total.

Acknowledgement. We thank Monique Laurent and Ronald de Wolf for bringing the topic of this paper to our attention. We also acknowledge support from ERC grant FOREFRONT (grant agreement no. 615640) funded by the European Research Council under the EU's 7th Framework Programme (FP7/20072013) and Ambizione grant PZ00P2 154779 Tight formulations of 0-1 problems funded by the Swiss National Science Foundation. Finally, we also thank the five anonymous referees for their constructive comments.

\section{References}

1. Michele Conforti, Gérard Cornuéjols, and Giacomo Zambelli. Extended formulations in combinatorial optimization. Ann. Oper. Res., 204:97-143, 2013.

2. Michele Conforti, Gérard Cornuéjols, and Giacomo Zambelli. Integer programming, volume 271 of Graduate Texts in Mathematics. Springer, Cham, 2014.

3. H. S. M. Coxeter. Projective geometry. Springer-Verlag, New York, 1994. Revised reprint of the second (1974) edition.

4. Jack Edmonds. Paths, trees, and flowers. Canad. J. Math., 17:449-467, 1965.

5. Jack Edmonds. Matroids and the greedy algorithm. Math. Programming, 1:127136, 1971.

6. Samuel Fiorini, Volker Kaibel, Kanstantsin Pashkovich, and Dirk Oliver Theis. Combinatorial bounds on nonnegative rank and extended formulations. Discrete Math., 313(1):67-83, 2013.

7. Samuel Fiorini, Serge Massar, Sebastian Pokutta, Hans Raj Tiwary, and Ronald de Wolf. Exponential lower bounds for polytopes in combinatorial optimization. J. ACM, 62(2):Art. 17, 23, 2015. 
8. Mika Göös, Rahul Jain, and Thomas Watson. Extension complexity of independent set polytopes. In Foundations of Computer Science (FOCS), 2016 IEEE 57th Annual Symposium on, pages 565-572. IEEE, 2016.

9. Volker Kaibel. Extended formulations in combinatorial optimization. arXiv preprint arXiv:1104.1023, 2011.

10. Kaveh Khoshkhah and Dirk Oliver Theis. Fooling sets and the spanning tree polytope. arXiv preprint arXiv:1701.00350, 2017.

11. R. Kipp Martin. Using separation algorithms to generate mixed integer model reformulations. Oper. Res. Lett., 10(3):119-128, 1991.

12. Thomas Rothvoß. The matching polytope has exponential extension complexity. In STOC'14-Proceedings of the 2014 ACM Symposium on Theory of Computing, pages 263-272. ACM, New York, 2014.

13. Tim Roughgarden. Communication complexity (for algorithm designers). arXiv preprint arXiv:1509.06257, 2015.

14. Alexander Schrijver. Combinatorial Optimization. Polyhedra and Efficiency. Springer, 2003.

15. Zsolt Tuza. Covering of graphs by complete bipartite subgraphs: complexity of $0-1$ matrices. Combinatorica, 4(1):111-116, 1984.

16. R.T. Wong. Integer programming formulations of the traveling salesman problem. In Proceedings of 1980 IEEE International Conference on Circuits and Computers, pages 149-152, 1980.

17. Mihalis Yannakakis. Expressing combinatorial optimization problems by linear programs. J. Comput. System Sci., 43(3):441-466, 1991. 